\title{
RELAÇÃO ENTRE ÍNDICE DE MASSA CORPORAL E PARTICIPAÇÃO NAS AULAS DE EDUCAÇÃO FÍSICA: UMA COMPARAÇÃO ENTRE ESCOLA PÚBLICA E PRIVADA
}

\author{
Eduardo Caputo \\ Universidade Federal de Pelotas, Pelotas, Rio Grande do Sul, Brasil. \\ Marcelo Cozzensa da Silva \\ Universidade Federal de Pelotas, Pelotas, Rio Grande do Sul, Brasil.
}

\begin{abstract}
Resumo
O presente estudo teve como objetivo verificar a relação entre participação nas aulas de Educação Física e o Índice de Massa Corporal (IMC) em escolares das redes de ensino pública e privada. A amostra foi composta por alunos da sexta série do Ensino Fundamental, 16 da rede pública e 26 da rede privada de ensino. A prevalência de sobrepeso e obesidade para alunos de escola pública e privada foram respectivamente: 43,7\%; 0,0\% e 34\%; 11,5\%. Foi encontrada diferença significativa entre o IMC dos alunos ativos quando comparado com os alunos inativos $\left(23,9 \pm 3,8 \mathrm{Kg} / \mathrm{m}^{2}\right.$ e 20,6 $\pm 3,1 \mathrm{Kg} / \mathrm{m}^{2}$, respectivamente; $\mathrm{p}=0,02)$. Foi possível concluir que os indivíduos mais ativos nas aulas apresentaram menor média de IMC. Palavras-chave: IMC - Obesidade - Sobrepeso - Educação Física.
\end{abstract}

\section{Introdução}

Segundo a Organização Mundial de Saúde (OMS), a obesidade é um problema epidemiológico de proporção mundial. Inatividade física associada com má alimentação e fatores genéticos configuram-se como os principais fatores associados para o ganho excessivo de gordura durante o crescimento em crianças e adolescentes. Adicionalmente, comportamentos como o uso de equipamentos eletrônicos e o tempo despendido em frente da televisão estão relacionados ao ganho de peso corporal (AMARAL; PALMA, 2001; MASCARENHAS et al., 2005).

A obesidade infantil se torna preocupante, posto que crianças e adolescentes obesos possuem maior risco de se tornarem adultos obesos. Complicações articulares, cardiovasculares e psicossociais, estão associadas ao excesso de peso corporal, assim como uma taxa de mortalidade aumentada (BALABAN; SILVA, 2001; MELLO; LUFT; MEYER, 2004; SABIA; SANTOS; RIBEIRO, 2004).

Os estudos realizados com escolares no Brasil apresentam uma grande variabilidade de resultados. $\mathrm{Na}$ cidade de Salvador a prevalência de obesidade foi de 30,0\% nas escolas particulares e 8,0\% nas públicas (LEÃO et al. 2003); em Feira de Santana observou-se prevalência de excesso de peso de 20,4\% em escolas particulares e 9,2\% em escolas públicas (OLIVEIRA; CERQUEIRA; OLIVEIRA 2003).

O ambiente escolar possui papel de destaque dentro dos hábitos alimentares e de prática de atividade física (COSTA; CINTRA; FISBERG, 2006). Os programas de 
Educação Física escolar devem, segundo Guedes e Guedes (1997), atender a duas metas prioritárias: a) promover experiências motoras que possam repercutir satisfatoriamente em direção a um melhor estado de saúde, procurando afastar ao máximo a possibilidade de aparecimento dos fatores de risco que contribuem para o surgimento de eventuais distúrbios orgânicos; e b) levar os educandos a assumirem atitudes positivas em relação à prática de atividades físicas para que se tornem ativos fisicamente não apenas na infância e na adolescência, mas também na idade adulta.

Em sua maioria, os programas de Educação Física escolar contemplam o aprendizado e o aperfeiçoamento de gestos técnicos de desportos como Futsal, Handebol, Basquete e Vôlei o que pode resultar em um afastamento de indivíduos pouco habilidosos. Segundo Guedes (1999), ao admitirmos que uma das principais metas da Educação Física escolar é promover um estilo de vida ativo, procurando melhorar o nível de qualidade de vida de nossa população, a inadequada utilização do esporte nos programas pode vir a atuar de forma inversa, o que pode, como consequência, levar ao ganho de peso excessivo. $\mathrm{O}$ presente estudo teve como objetivo verificar a relação entre participação nas aulas de Educação Física e o Indice de Massa Corporal (IMC) em escolares das redes de ensino pública e privada.

\section{Metodologia}

O presente estudo caracteriza-se por ser do tipo observacional de corte transversal. O estudo foi realizado em duas escolas da cidade de Pelotas-RS, uma pertencente à rede pública que atende principalmente uma população de nível socioeconômico reduzido, a segunda faz parte da rede privada e atende de forma preferencial uma população de nível socioeconômico alto.

Os responsáveis assinaram um Termo de Consentimento Livre e Esclarecido, autorizando a participação do aluno, e o projeto de pesquisa foi aprovado pelo Comitê de Ética em Pesquisa com seres humanos da ESEF/UFPel sob o número de protocolo 052.

\section{Amostra}

A seleção da amostra foi por conveniência, composta por duas turmas de $6^{\mathrm{a}}$ série do Ensino Fundamental. A turma referente à escola pública foi composta por 16 alunos, sendo 10 do sexo masculino e 6 do feminino. A turma correspondente à escola privada possuía 26 alunos, 12 do sexo masculino e 14 do feminino.

Aqueles indivíduos que apresentaram uma abstenção maior que $75 \%$ nas aulas de Educação Física ao longo do semestre foram excluídos do estudo.

\section{Peso, estatura e IMC}

O peso corporal foi mensurado com o auxílio de uma balança da marca Filizola com precisão de $0,1 \mathrm{Kg}$. No momento da medida, os indivíduos estavam com os pés descalços e vestiam apenas bermuda e camiseta. Para a determinação da estatura foi utilizada uma fita métrica flexível colocada em uma parede lisa. No momento da medida, os sujeitos encontravam-se descalços. 
O Índice de Massa Corporal (IMC) foi calculado a partir da equação massa corporal em quilos dividido pela altura em metros elevado ao quadrado. Sobrepeso e obesidade foram definidos com IMC igual ou superior aos percentis 85 e 95 para idade e sexo (COLE et al., 2000).

Participação nas aulas de Educação Física

A participação nas aulas de Educação Física foi estabelecida por meio de dois critérios: a percepção do professor em relação ao envolvimento prático do aluno nas aulas; e a frequência mínima de $75 \%$ da quantidade total de aulas no semestre. Para ser considerado ativo nas aulas, o aluno deveria enquadrar-se nos critérios descritos acima.

\section{Análise estatística}

Para a análise utilizou-se o programa STATA 10.0. Foi utilizado o teste de Wilcoxon não pareado para comparação entre médias dos grupos estudados. Para verificar a relação entre participação nas aulas de Educação Física e IMC foi utilizado ANOVA.

\section{Resultados}

A média de idade da amostra foi de $12 \pm 1,4$ anos, sendo que os alunos da escola pública apresentaram média de idade de $12,4 \pm 0,7$ anos, e os da particular 11,7 $\pm 1,0$ anos. Com relação ao peso corporal e a estatura, as médias encontradas foram respectivamente de 52,2 $\pm 25,5 \mathrm{Kg}$ e $156,5 \pm 14,1 \mathrm{~cm}$. Quando analisadas separadamente, a escola pública e privada apresentaram os seguintes valores, respectivamente: 57,6 \pm $19,7 \mathrm{~kg}$ e $164,1 \pm 13,8 \mathrm{~cm} ; 49 \pm 24 \mathrm{Kg}$ e $151,8 \pm 12,7 \mathrm{~cm}$.

O IMC médio da amostra foi de $21,2 \pm 9,8 \mathrm{Kg} / \mathrm{m}^{2}$, sendo de $21,2 \pm 2,7 \mathrm{Kg} / \mathrm{m}^{2}$ para os alunos da escola pública e $21,2 \pm 3,9 \mathrm{Kg} / \mathrm{m}^{2}$ para os da particular $(\mathrm{p}>0,05)$. Os meninos apresentaram IMC estatisticamente superior ao das meninas $(21,6 \pm 2,3$ $\mathrm{Kg} / \mathrm{m}^{2}$ vs $20,9 \pm 4,7 \mathrm{Kg} / \mathrm{m}^{2}$; $\left.\mathrm{p}<0,05\right)$.

Quando classificados com relação à idade, não houve diferença entre o IMC e o nível de participação nas aulas de Educação Física ( $p>0,05)$. Os alunos que demonstram ser inativos nas aulas de Educação Física apresentaram média de IMC de 23,9 $\pm 3,8 \mathrm{Kg} / \mathrm{m}^{2}$ contra $20,6 \pm 3,1 \mathrm{Kg} / \mathrm{m}^{2}$ dos alunos considerados ativos $(\mathrm{p}=0,02)$.

Os dados referentes à classificação do IMC, com relação à idade, são apresentados na Tabela 1.

Tabela 1. Prevalência nas categorias relativas ao índice de massa corporal dos alunos de escola pública e privada da cidade de Pelotas/RS

\begin{tabular}{|c|c|c|c|}
\hline Escola & Eutófrico & Sobrepeso & Obesidade \\
\hline Pública & $56,2 \%(n=9)$ & $43,7 \%(n=7)$ & $0,0 \%(n=0)$ \\
\hline Privada & $54,5 \%(n=14)$ & $34,0 \%(n=9)$ & $11,5 \%(n=3)$ \\
\hline Total & $55,0 \%(n=23)$ & $38,0 \%(n=16)$ & $7,0 \%(n=3)$ \\
\hline
\end{tabular}


Com relação à participação nas aulas de Educação Física, 19,0\% da amostra total demonstrou ser inativa. Quando separadas, escola pública de escola privada, esta última apresentou os seguintes valores, respectivamente: 18,75\% e 19,23\% ( $\mathrm{p}=0,65)$ (Tabela 2).

Tabela 2. Prevalência de participação nas aulas de Educação Física

\begin{tabular}{|c|c|c|}
\hline Escola & Ativos & Inativos \\
\hline Pública & $81,2 \%(\mathrm{n}=13)$ & $18,7 \%(\mathrm{n}=3)$ \\
\hline Privada & $80,8 \%(\mathrm{n}=21)$ & $19,2 \%(\mathrm{n}=5)$ \\
\hline
\end{tabular}

\section{Discussão}

Quando os valores de IMC foram corrigidos e classificados por idade, 43,7\% e $34,0 \%$ dos alunos da rede pública e privada, respectivamente, apresentaram sobrepeso e apenas na rede privada foram encontrados indivíduos que apresentaram obesidade (11,5\%). Ao estudar escolares da rede pública e privada da cidade de Feira de Santana, Oliveira, Cerqueira e Oliveira, (2003) encontraram que, respectivamente, 6,5\% e 13,4\% dos alunos apresentavam sobrepeso. Leão et al. (2006) encontraram uma prevalência de obesidade de 30\% em escolares da rede privada e 8,2\% em escolares da rede pública da cidade de Salvador.

Nossos achados evidenciam que os alunos que demonstraram ser mais ativos nas aulas de Educação Física apresentaram IMC médio significativamente maior que os alunos inativos. Aquino Jr., Barreto e Okada, (2008) não encontraram diferença na participação de crianças com sobrepeso e peso normal nas aulas de Educação Física. Os autores afirmam que uma possível explicação seria a prática reduzida de atividade física fora do âmbito escolar e uma alimentação rica em alimentos gordurosos.

O aumento no consumo de alimentos gordurosos, com alta densidade energética e a diminuição na prática de exercícios físicos são os dois principais fatores comportamentais que podem colaborar para o aumento da prevalência da obesidade (HILL; TROWBRIDGE, 1998). Celestrino e Costa (2006) colocam que a reeducação alimentar e a prática de atividade física fomentada no âmbito escolar são de vital importância, pois podem contribuir para a profilaxia da obesidade infanto-juvenil, evitando que complicações de ordem fisiológica e psicossociais provocadas por essa enfermidade interfiram na qualidade de vida dos indivíduos, reduzindo as possibilidades desses tornarem-se adultos obesos.

Guedes e Guedes (1997) ressaltam a importância de tornar as aulas de Educação Física mais atrativas a fim de conseguir maior adesão dos alunos e torná-los adultos ativos. Ainda descrevem que a utilização das quatro modalidades esportivas tradicionais (Futsal, Handebol, Basquete e Vôlei) pode ter uma influência negativa na prática de um estilo de vida ativo fora do ambiente escolar, pois muitos não apresentam a mesma facilidade quanto ao domínio das habilidades específicas que a prática exige, levando a um desinteresse por esse tipo de atividade. 
Com o passar dos anos, os jovens passam a demonstrar insatisfação com a oferta de atividades desportivas, com o nível de competição e divertimento que existe nas aulas de Educação Física. Este efeito progressivo parece ocorrer a partir do $4^{\circ}$ ano de escolaridade. Isso demonstra a importância que possui o prazer e o divertimento na disciplina de Educação Física nos hábitos futuros de atividade física dos adolescentes (GODIN; SHEPHARD, 1986; SALLIS et al. 1999).

Quando comparados em relação ao sexo, os meninos apresentaram IMC estatisticamente superior ao das meninas $\left(21,6 \pm 2,3 \mathrm{Kg} / \mathrm{m}^{2}\right.$ vs $20,9 \pm 4,7 \mathrm{Kg} / \mathrm{m}^{2}$; p<0,05). Meninos e meninas apresentam diferenças quanto à composição corporal independente do estágio de maturação sexual em que se encontram. Nas meninas, se observa um aumento gradual nos estoques de gordura corporal durante a adolescência, enquanto, nos meninos, ocorre um ganho mais acentuado de massa muscular, na fase de velocidade máxima de crescimento (COLLI, 1991).

Algumas limitações do estudo devem ser apontadas. O presente trabalho apresenta delineamento transversal, o que pode acarretar em viés de causalidade reversa. Outra limitação do estudo é o fato de não terem sido coletados dados referentes ao nível de maturação sexual e atividade física realizada fora da escola. Além disso, o tamanho amostral dificulta a possibilidade de verificação de algumas associações por acarretar falta de poder estatístico (SIERVOGEL et al., 2003; SEABRA et al., 2008).

A criação de ambientes apropriados a práticas de atividades físicas bem como o planejamento de aulas atrativas e que atendam os anseios dos alunos são fundamentais para a manutenção e desenvolvimento de hábitos saudáveis de vida. Dessa forma, o ambiente escolar é de fundamental importância tanto em relação à prática de exercícios físicos quanto de uma alimentação saudável.

Conclui-se que não houve diferenças com relação à participação nas aulas de Educação Física entre os estudantes das redes de ensino pública e privada e que os indivíduos que demonstraram ser mais ativos nas aulas apresentaram menor IMC que os inativos. A realização de estudos abrangendo maior tamanho amostral de indivíduos de ambos os sexos com verificação do nível de maturação sexual e das atividades físicas realizadas no tempo de lazer devem ser consideradas na realização de novos estudos sobre o tema.

\title{
Relation between body mass index and participation in Physical Education classes: a comparison between public and private school
}

\begin{abstract}
Aim: This study aimed to investigate the relation between participation in Physical Education classes and body mass index (BMI) in students of public and private school. Materials and Methods: The sample consisted of students from the sixth grade of elementary school, 16 of public and 26 of private schools. Results: The prevalence of overweight and obesity in students of public and private school are respectively: $43,7 \% ; 0,0 \%$ e $34 \% ; 11,5 \%$. We found significant differences between BMI of active students compared to sedentary one $\left(23.9 \pm 3.8 \mathrm{~kg} / \mathrm{m}^{2}\right.$ and $20.6 \pm 3.1 \mathrm{~kg}$ $/ \mathrm{m}^{2}$ respectively, $\left.\mathrm{p}=0.02\right)$. Conclusion: It was possible to conclude that individuals more active in class had lower BMI.
\end{abstract}

Keywords: BMI - Obesity - Overweight - Physical Education. 
Relación entre el índice de masa corporal y la participación en clases de Educación Física: una comparación entre la escuela pública y la privada

\section{Resumen}

Objetivo: el presente estudio tuvo como objetivo verificar la relación entre participación en las clases de Educación Física y el Índice de Masa Corporal (IMC) en escolares de las redes de enseñanza pública y privada. La muestra se compuso con alumnos de sexta serie de la Enseñanza Fundamental, 16 de la red pública y 26 de la red privada de enseñanza. La prevalencia de sobrepeso y obesidad para alumnos de escuela pública y privada fueron respectivamente: $43,7 \% ; 0,0 \%$ e $34 \% ; 11,5 \%$. Se encontró una diferencia importante entre el IMC de los alumnos activos cuando se compara con los alumnos inactivos (23,9 \pm $3,8 \mathrm{Kg} / \mathrm{m}^{2}$ y $20,6 \pm 3,1 \mathrm{Kg} / \mathrm{m}^{2}$, respectivamente; $\mathrm{p}=0,02$ ). Fue posible concluir que los individuos más activos en las clases presentaron un promedio menor de IMC.

Palabras clave: IMC - Obesidad - Sobrepeso - Educación Física.

\section{Referências}

AQUINO JR, A. E.; BARRETO, S. M.G.; OKADA, G. T. Relação e envolvimento de crianças obesas em aulas de Educação Física em escola municipal de Educação Infantil (EMEI) de São Carlos-SP. Revista Brasileira de Educação Física, Esporte, Lazer e Dança. v. 3, n. 1, p.1-9, 2008.

AMARAL, A. P. de A. P.; PALMA, A. Perfil epidemiológico da obesidade em crianças: relação entre televisão, atividade física e obesidade. Revista Brasileira Ciência e Movimento, Brasília, v. 9, n. 4, p. 19-24, 2001.

BALABAN, G.; SILVA, G. A. P. Prevalência de sobrepeso e obesidade em crianças e adolescentes de uma escola da rede privada de Recife. Jornal de Pediatria. v. 77, n. 2, p. 96-100, 2001.

CELESTRINO, J. O.; COSTA, A. S. A prática de atividade física entre escolares com sobrepeso e obesidade. Revista Mackenzie de Educação Física e Esporte. v. 5, p. $47-54 ; 2006$.

COLE, T. J. et al. Establishing a standard definition for child overweight and obesity worldwide: international survey. BMJ. v. 320, p.1-6, 2000.

COLLI, A. S. Crescimento e desenvolvimento físico do adolescente. In: MAAKAROUN, M. F, SOUZA, R. P., CRUZ, A. R., (eds). Tratado de adolescência: um estudo multidisciplinar. Rio de Janeiro: Cultural Médica, 1991. p.243-257.

COSTA, R. F.; CINTRA, I. de P.; FISBERG, M. Prevalência de sobrepeso e obesidade em escolares da cidade de Santos, SP. Arq Bras Endocrinol Metab. v. 50, n. 1, p. 60-67, 2006.

GODIN, G.; SHEPHARD, R. Psychosocial factors influencing intentions to exercise of young students from grades 7 to 9. Research Quarterly for Exercise and Sport, v. 57, n. 1, p. 41-52, 1986.

GUEDES, D. P. Educação para a saúde mediante programas de Educação Física escolar. Motriz. v. 5, n. 1, p. 10-14, 1999. 
GUEDES, J. E. R. P.; GUEDES, D. P. Características dos programas de Educação Física escolar. Revista Paulista de Educação Físisca, São Paulo, v. 11, n. 1, p. 49-62, 1997.

HILL, J. O.; TROWBRIDGE, F. L. Childhood obesity: future directions and research priorities. Pediatrics, n. 101, p. 570-574, 1998.

LEÃO, L. S. C. et al. Prevalência de obesidade em escolares de Salvador, Bahia. Arq Bras Endocrinol Metab. v. 47, n. 2, p. 151-157, 2003.

MASCARENHAS, L. P. G. et al. Relação entre diferentes índices de atividade física e preditores de adiposidade em adolescentes de ambos os sexos. Rev Bras Med Esporte, v. 11, n. 4, p.214-218, 2005.

MELLO, E. D.; LUFT, V. C.; MEYER, F. Obesidade infantil: como podemos ser eficazes? Jornal de Pediatria. v. 80, n. 3, p. 173-182, 2004.

OLIVEIRA, A. M. A.; CERQUEIRA, E. M. M.; OLIVEIRA, A. C. Prevalência de sobrepeso e obesidade infantil na cidade de Feira de Santana-BA: detecção na família x diagnóstico clínico. Jornal de Pediatria, Rio de Janeiro, v. 79, n. 4, p. 325-328, 2003. ORGANIZAÇÃO MUNDIAL DA SAÚDE. Obesidad y sobrepeso. n. 311, set. 2006 Disponível em: <http://www.who.int/mediacentre/factsheets/fs311/es/> . Acesso em: out. 2008.

SABIA, R. V.; SANTOS, J.E.; RIBEIRO, R.P.P.Efeito da atividade física associada à orientação alimentar em adolescentes obesos: comparação entre o exercício aeróbio e anaeróbio. Revista Brasileira de Medicina do Esporte, v. 10, n. 5, p. 349-355, 2004.

SALLIS,J. F. et al.Predictors of change in children's physical activity over 20 months: variations by gender and level of adiposity. Am J Prev Med, v. 16, n. 3, p. 222-229, 1999.

SEABRA, A. F. et al. Determinantes biológicos e sócio-culturais associados à prática de atividade física de adolescentes. Cad Saúde Pública, v. 24, n. 4, p. 721-736, 2008.

SIERVOGEL, R. M. et al. Puberty and body composition. Horm Res, v. 60, Suppl. 1, p. 36-45, 2003.

Recebido em: 20/07/2009

Revisado em: 02/10/2009

Aprovado em: 12/11/2009

Endereço para correspondência

Marcelo Cozzensa da Silva

Universidade Federal de Pelotas

Escola Superior de Educação Fisica

Rua Luiz de Camões, 625 - Cohab Tablada

CEP: 96055-630 - Pelotas, RS - Brasil

E-mail:cozzensa@terra.com.br 